\title{
Involutions of Double Affine Hecke Algebras
}

\author{
BOGDAN ION \\ Department of Mathematics, Princeton University, Princeton, NJ-08544, U.S.A. \\ e-mail:bogdan@math.princeton.edu
}

(Received: 16 November 2001; accepted in final form 22 May 2002)

\begin{abstract}
We prove the existence of an involution on double affine Hecke algebras. This involution plays a central role in the theory of Macdonald polynomials, being responsible for a Fourier type duality and allowing the construction of affine intertwiners.
\end{abstract}

Mathematics Subject Classifications (2000). Primary: 20C08, 33D52; Secondary: 33D80.

Key words. Artin group, fundamental group, Hecke algebra, Macdonald polynomials, regular orbits space.

\section{Introduction}

The double affine Hecke algebras were introduced by Cherednik [C1], [C2] in connection with affine quantum Knizhnik-Zamolodchikov equations and eigenvalue problems of Macdonald type. As a general principle one can associate to any type of root system (finite, affine, elliptic) a Weyl group, an Artin group and a Hecke algebra. Along these lines, double affine Hecke algebras are associated with a certain class of elliptic root systems. Section 1 contains the definitions and relevant results about double affine Weyl groups, their Artin groups and their Hecke algebras as well as a topological interpretation of affine Artin groups due to H. van der Lek.

One of the key features of double affine Hecke algebras is that they contain two commutative subalgebras. Our main result roughly states that there exists a pairing between the root systems in question such that their corresponding Weyl groups, Artin groups and Hecke algebras are canonically isomorphic in such a way that for the Hecke algebras the roles of the above mentioned commutative subalgebras is interchanged. Section 2 contains the precise statements of these results.

This involution of the double affine Hecke algebra plays a central role in the theory of Macdonald polynomials. For example, Cherednik [C3] used it to define the difference Fourier transform which is the crucial ingredient in his proof of Macdonald's evaluation-duality conjecture. The evaluation-duality phenomenon was first noticed and proved by Koornwinder in the $A_{n}$ case [Ko] [M1, Chapter IV, Section 6] and then formulated as conjecture for all root systems by Macdonald. This phenomenon was recognized by Cherednik as a Fourier type duality: the interchange of variable and spectral parameter. The transform responsible for this duality is the difference Fourier transform. As another consequence of the existence of such 
canonical isomorphisms let us mention the construction of affine intertwiners. The importance of the intertwiners was first understood by Knop and Sahi [Kn, KS, S1] for $\mathrm{GL}_{n}$ and then by Cherednik [C4] in the general (reduced) case.

The proof relies on the topological interpretation of extended Artin groups due to H. van der Lek [L1, L2]. This relates the double affine Artin groups and the fundamental groups of certain complex hyper-plane complements. Section 3 reveals this connection and establishes the canonical isomorphism at the level of Artin groups. The advantage of this point of view is that the isomorphism of the fundamental groups, and consequently of the Artin groups, is induced from a very simple transformation of the space of complex hyperplane complements: the interchange of real and imaginary parts. Section 4 finishes the proof of the existence of the canonical isomorphism at the level of Hecke algebras.

The statements of our main result as well as the connection between the double affine Artin groups and certain fundamental groups were announced in Theorem 2.2 and Theorem 2.4 of [C1]. Macdonald [M5, Chapter III, Sections 3.5-3.7] also checked the existence of the involution using a case-by-case analysis.

\section{Preliminaries}

\subsection{AFFINE AND DOUBLE AFFINE WEYL GROUPS}

For the most part we shall adhere to the notation in [K]. Let $A=\left(a_{j k}\right)_{0 \leqslant j, k \leqslant n}$ be an irreducible affine Cartan matrix, $S(A)$ the Dynkin diagram and $\left(a_{0}, \ldots, a_{n}\right)$ the numerical labels of $S(A)$ in Table Aff from $[\mathrm{K}]$, p. 48-49. We denote by $\left(a_{0}^{\vee}, \ldots, a_{n}^{\vee}\right)$ the labels of the Dynkin diagram $S\left(A^{t}\right)$ of the dual diagram which is obtained from $S(A)$ by reversing the direction of all arrows and keeping the same enumeration of the vertices. Let $\left(\mathfrak{h}, R, R^{\vee}\right)$ be a realization of $A$ and let $\left(\mathfrak{h}, R, R^{\vee}\right)$ be the associated finite root system (which is a realization of the Cartan matrix $\left.\stackrel{\circ}{A}=\left(a_{j k}\right)_{1 \leqslant j, k \leqslant n}\right)$. If we denote by $\left\{\alpha_{j}\right\}_{0 \leqslant j \leqslant n}$ a basis of $R$ such that $\left\{\alpha_{j}\right\}_{1 \leqslant j \leqslant n}$ is a basis of $\stackrel{\circ}{R}$ we have the following description

$$
\mathfrak{h}^{*}=\mathfrak{h}^{*}+\mathbb{R} \delta+\mathbb{R} \Lambda_{0}
$$

where $\delta=\sum_{j=0}^{n} a_{j} \alpha_{j}$. The vector space $\mathfrak{h}^{*}$ has a canonical scalar product defined as follows

$$
\left(\alpha_{j}, \alpha_{k}\right):=d_{j}^{-1} a_{j k}, \quad\left(\Lambda_{0}, \alpha_{j}\right):=\delta_{j, 0} a_{0}^{-1} \quad \text { and } \quad\left(\Lambda_{0}, \Lambda_{0}\right):=0,
$$

with $d_{j}:=a_{j} a_{j}^{\vee-1}$ and $\delta_{j, 0}$ Kronecker's delta. The simple coroots are $\left\{\alpha_{j}^{\vee}:=\right.$ $\left.d_{j} \alpha_{j}\right\}_{0 \leqslant j \leqslant n}$. Denote by $Q=\bigoplus_{j=1}^{n} Z \alpha_{j}$ and $Q^{\vee}=\bigoplus_{j=1}^{n} Z \alpha_{j}^{\vee}$ the root lattice, respectively the coroot lattice of $\stackrel{\circ}{R}$ and by $Q=\bigoplus_{j=0}^{n} Z \alpha_{j}=\stackrel{Q}{Q} \bigoplus Z \delta$ the root lattice of $R$.

Given $\alpha \in R, x \in \mathfrak{h}^{*}$ let

$$
s_{\alpha}(x):=x-\frac{2(x, \alpha)}{(\alpha, \alpha)} \alpha .
$$


The affine Weyl group $W$ is the subgroup of $\operatorname{GL}\left(\mathfrak{G}^{*}\right)$ generated by all $s_{\alpha}$ (the simple reflexions $s_{j}=s_{\alpha_{j}}$ are enough). The finite Weyl group $\stackrel{\circ}{W}$ is the subgroup generated by $s_{1}, \ldots, s_{n}$. Both the finite and the affine Weyl group are Coxeter groups and they can be abstractly defined as generated by $s_{1}, \ldots, s_{n}$, respectively $s_{0}, \ldots, s_{n}$, and some relations. These relations are called Coxeter relations and they are of two types: the reflection relations $s_{j}^{2}=1$ and the braid relations (see $[\mathrm{H}]$ for details).

The double affine Weyl group $\widetilde{W}$ is defined to be the semidirect product $W \ltimes Q$ of the affine Weyl group and the lattice $Q$ (regarded as an abelian group with elements $\tau_{\beta}$, where $\beta$ is a root), the affine Weyl group acting on the root lattice as follows

$$
w \tau_{\beta} w^{-1}=\tau_{w(\beta)} .
$$

This group is the hyperbolic extension of an elliptic Weyl group which is by definition the Weyl group associated with an elliptic root system (see [ST] for definitions). It also has a presentation with generators and relations (elliptic Coxeter relations). We refer the reader to $[\mathrm{ST}]$ for the details.

The affine Weyl group $W$ can also be presented as a semidirect product in the following way. For $1 \leqslant j \leqslant n$, let $e_{j}=\max \left\{a_{0}^{-1}, d_{j}\right\}$. Recall that $a_{0}=1$ in all cases except for $A=A_{2 n}^{(2)}$, where $a_{0}=2$. Denote by $M$ the lattice generated by $\left\{A_{j}=e_{j} \alpha_{j}\right\}_{1 \leqslant j \leqslant n}$. Then $W$ is the semidirect product of $W$ and the lattice $M$ (regarded as an abelian group with elements $\lambda_{\mu}$, where $\mu$ is in $M$ ), the finite Weyl group acting on the lattice $M$ as follows

$$
w \lambda_{\mu} w^{-1}=\lambda_{w(\mu)} .
$$

Let us remark that the numbers $e_{j}$ depend only on the length of the corresponding simple root. Therefore, we will write $e_{s}, e_{l}$ for $e_{j}$ if the corresponding simple root is short, respectively long. The number

$$
p:=e_{s}
$$

will play an important role. It is easy to see that $p \in\{1,2,3\}$ (it represents in fact the affine type of $A^{t}$ ). If $\beta \in \stackrel{\circ}{R}, e_{\beta}$ will denote $e_{s}$ or $e_{l}$ depending on the length of $\beta$. With this notation define

$$
A_{\beta}:=e_{\beta} \beta \quad \text { and } \quad A_{\beta}^{\vee}:=e_{\beta}^{-1} \beta^{\vee} .
$$

For $r$ a real number, $\mathfrak{h}_{r}^{*}=\{x \in \mathfrak{h} ;(x, \delta)=r\}$ is the level $r$ of $\mathfrak{h}^{*}$. We have

$$
\mathfrak{h}_{r}^{*}=\mathfrak{h}_{0}^{*}+r \Lambda_{0}=\mathfrak{h}^{*}+\mathbb{R} \delta+r \Lambda_{0}
$$

The action of $W$ preserves each $\mathfrak{h}_{r}^{*}$ and we can identify each $\mathfrak{h}_{r}^{*}$ canonically with $\mathfrak{h}_{0}^{*}$ and obtain an (affine) action of $W$ on $\mathfrak{h}_{0}^{*}$. For example, the level zero action of $s_{0}$ and $\lambda_{\mu}$ on $\mathfrak{h}_{0}^{*}$ is

$$
\begin{aligned}
& s_{0}(x)=s_{\theta}(x)+(x, \theta) \delta, \\
& \lambda_{\mu}(x)=x-(x, \mu) \delta,
\end{aligned}
$$


and the level one affine action of the same elements on $\mathfrak{h}^{*}$ is

$$
s_{0}(x)=s_{\theta}(x)+a_{0}^{-1} \theta, \quad \lambda_{\mu}(x)=x+\mu,
$$

where we denoted by $\theta=\delta-a_{0} \alpha_{0}$.

\subsection{ARTIN GROUPS AND HECKE ALGEBRAS}

To any Coxeter group we can associate its Artin group, the group defined with the same generators which satisfy only the braid relations (that is, forgetting the reflexion relations). The finite and affine Weyl groups are Coxeter groups; we will make precise the definition of the Artin groups in these cases. First let us make the following convention

For the rest of the paper we assume our irreducible affine Cartan matrix to be of any type except $A_{2 n}^{(2)}$.

Note that the corresponding definitions and results of this paper (at the level of Hecke algebras) for the case omitted can be found in [S2].

DEFINITION 1.1. With the notation above define

(i) the finite Artin group $\mathcal{A}_{W}$ as the group generated by elements $T_{1}, \ldots, T_{n}$ satisfying the same braid relations as the reflexions $s_{1}, \ldots, s_{n}$;

(ii) the affine Artin group $\mathcal{A}_{W}$ as the group generated by the elements $T_{0}, \ldots, T_{n}$ satisfying the same braid relations as the reflexions $s_{0}, \ldots, s_{n}$.

From the definition it is clear that the finite Artin group can be realized as a subgroup inside the affine Artin group. To define the Hecke algebras, we introduce a field $\mathbb{F}$ (of parameters) as follows: fix indeterminates $q$ and $t_{0}, \ldots, t_{n}$ such that $t_{j}=t_{k}$ if and only if $d_{j}=d_{k}$; let $m$ be the lowest common denominator of the rational numbers $\left\{\left(\alpha_{j}, \lambda_{k}\right) \mid 1 \leqslant j, k \leqslant n\right\}$, and let $\mathrm{F}$ denote the field of rational functions in $q^{\frac{1}{m}}$ and $t_{j}^{\frac{1}{2}}$. Because in our case there are at most two different root lengths we will also use the notation $t_{l}, t_{s}$ for $t_{j}$ if the corresponding simple root is long, respectively short.

For further use we also introduce the following lattices $\mathcal{M}_{Y}:=\left\{Y_{\mu} ; \mu \in M\right\}$ and $\mathcal{Q}_{X}:=\left\{X_{\beta} ; \beta \in Q\right\}$. We will use the same notation for their group F-algebras.

DEFINITION 1.2. The finite Hecke algebra $\mathcal{H}_{W}$ is the quotient of the group Falgebra of the finite Artin group by the relations

$$
T_{j}-T_{j}^{-1}=t_{j}^{\frac{1}{2}}-t_{j}^{-\frac{1}{2}}
$$

for $1 \leqslant j \leqslant n$.

Recall that the affine Weyl group also has a second presentation, as a semidirect product. There is a corresponding description of the affine Artin group (and consequently of the affine Hecke algebra) due to van der Lek [L1], Lusztig [Lu] and Bernstein (unpublished). 
PROPOSITION 1.3. The affine Artin group $\mathcal{A}_{W}$ is generated by the finite Artin group and the lattice $\mathcal{M}_{Y}$ such that the following relations are satisfied for all $1 \leqslant j \leqslant n$

(i) $T_{j} Y_{\mu}=Y_{\mu} T_{j}$ if $\left(\mu, A_{j}^{\vee}\right)=0$,

(ii) $T_{j} Y_{\mu} T_{j}=Y_{s_{j}(\mu)}$ if $\left(\mu, A_{j}^{\vee}\right)=1$.

Remark 1.4. In this description $Y_{\mu}=T_{\lambda_{\mu}}$ for $\mu$ any anti-dominant element of the lattice $M$. For example $Y_{-\theta}=T_{s_{\theta}} T_{0}$.

DEFINITION 1.5. The affine Hecke algebra $\mathcal{H}_{W}$ is the quotient of the group F-algebra of the affine Artin group by the relations

$$
\begin{aligned}
& T_{j}-T_{j}^{-1}=t_{j}^{\frac{1}{2}}-t_{j}^{-\frac{1}{2}}, \quad \text { for all } 0 \leqslant j \leqslant n, \\
& Y_{-A_{j}} T_{j}^{-1}-T_{j} Y_{A_{j}}=t_{j}^{\frac{1}{2}}-t_{j}^{-\frac{1}{2}}, \quad \text { for all } 1 \leqslant j \leqslant n .
\end{aligned}
$$

The elements $T_{1}, \ldots, T_{n}$ generate the finite Hecke algebra $\mathcal{H}_{W}$. There are natural bases of $\mathcal{H}_{W}$ and $\mathcal{H}_{W}:\left\{T_{w}\right\}_{w}$ indexed by $w$ in $W$ and in $\stackrel{\circ}{W}$ respectively, where $T_{w}=T_{j_{l}}, \ldots, T_{j_{1}}$ if $w=s_{j_{l}}, \ldots, s_{j_{1}}$ is a reduced expression of $w$ in terms of simple reflexions.

We must remark that the objects we define here are not the objects traditionally used in the literature: extended affine Artin groups and Hecke algebras. Nevertheless, their structure is completely similar. Regarding the definition of Hecke algebras we note that the relations (3) and (2) are conjugate inside the extended affine Artin group, but not always inside the affine Artin group. Therefore, only the relations (2) have to be required in the definition of extended affine Hecke algebras.

As before, the affine Hecke algebra has a presentation in terms of the finite Hecke algebra and the group algebra $\mathcal{M}_{Y}$ (see [Lu] for the proof).

PROPOSITION 1.6. The affine Hecke algebra $\mathcal{H}_{W}$ is generated by the finite Hecke algebra and the group algebra $\mathcal{M}_{Y}$ such that the following relation is satisfied for any $\mu$ in the lattice $M$ and any $1 \leqslant j \leqslant n$ :

$$
Y_{\mu} T_{j}-T_{j} Y_{s_{j}(\mu)}=\left(t_{j}^{\frac{1}{2}}-t_{j}^{-\frac{1}{2}}\right) \frac{Y_{\mu}-Y_{s_{j}(\mu)}}{1-Y_{A_{j}}} .
$$

Remark 1.7. An immediate consequence of Proposition 1.6 is that the relation

$$
T_{0}-T_{0}^{-1}=t_{0}^{\frac{1}{2}}-t_{0}^{-\frac{1}{2}}
$$

it is contained in the ideal generated by all the other relations defining the affine Hecke algebra.

The definition of the double affine Artin group and Hecke algebra is due to Cherednik (see, for example, $[\mathrm{Cl}]$ ). 
DEFINITION 1.8. The double affine Artin group $\mathcal{A}_{\widetilde{W}}$ is generated by the affine Artin group $\mathcal{A}_{W}$, the lattice $\mathcal{Q}_{X}$ and the element $X_{\delta}$ such the following relations are satisfied for all $0 \leqslant i \leqslant n$

(i) $X_{\delta}$ is central,

(ii) $T_{j} X_{\beta}=X_{\beta} T_{j}$ if $\left(\beta, \alpha_{j}^{\vee}\right)=0$,

(iii) $T_{j} X_{\beta} T_{j}=X_{s_{j}(\beta)}$ if $\left(\beta, \alpha_{j}^{\vee}\right)=-1$.

The double affine Weyl group is not a Coxeter group, but a generalized Coxeter group (in the sense of Saito and Takebayashi, see [ST]) and we can define the associated Artin group in the same way as for a Coxeter group (i.e. by keeping the generalized braid relations and forgetting the reflexion relations). For some elliptic Artin groups the equivalence of the two definitions is estabilished in [T].

DEFINITION 1.9. The double affine Hecke algebra $\mathcal{H}_{\widetilde{W}}$ is the quotient of the group $\mathbb{F}$-algebra of the double affine Artin group by the relations

$$
\begin{aligned}
T_{j}-T_{j}^{-1}=t_{j}^{\frac{1}{2}}-t_{j}^{-\frac{1}{2}}, & \text { for all } 1 \leqslant j \leqslant n, \\
Y_{-A_{j}} T_{j}^{-1}-T_{j} Y_{A_{j}}=t_{j}^{\frac{1}{2}}-t_{j}^{-\frac{1}{2}}, & \text { for all } 1 \leqslant j \leqslant n, \\
T_{j}^{-1} X_{\alpha_{j}}-X_{-\alpha_{j}} T_{j}=t_{j}^{\frac{1}{2}}-t_{j}^{-\frac{1}{2}}, & \text { for all } 1 \leqslant j \leqslant n, \\
T_{0}^{-1} X_{\alpha_{0}}-X_{-\alpha_{0}} T_{0}=t_{0}^{\frac{1}{2}}-t_{0}^{-\frac{1}{2}}, &
\end{aligned}
$$

and by

$$
X_{\delta}=q^{-1} .
$$

As before, $\mathcal{H}_{\widetilde{W}}$ can be described in terms of $\mathcal{H}_{W}$ and $\mathcal{Q}_{X}$.

PROPOSITION 1.10 ([C1]). The double affine Hecke algebra $\mathcal{H}_{\widetilde{W}}$ is the $\mathbb{F}$-algebra generated by the affine Hecke algebra $\mathcal{H}_{W}$ and the group algebra $\mathcal{Q}_{X}$ such that, with the notation $X_{\delta}=q^{-1}$, the following relation is satisfied for any $\beta$ in the root lattice and any $0 \leqslant j \leqslant n$ :

$$
T_{j} X_{\beta}-X_{s_{j}(\beta)} T_{j}=\left(t_{j}^{\frac{1}{2}}-t_{j}^{-\frac{1}{2}}\right) \frac{X_{\beta}-X_{S_{j}(\beta)}}{1-X_{-\alpha_{j}}} .
$$

The proof is completely similar to the proof of Proposition 1.6.

\subsection{SOME RESULTS OF VAN DER LEK}

Inspired by the techniques introduced in [D], H. van der Lek developed in [L2] a machinery which allows one to compute fundamental groups of complex hyperplane complements. As an application he realized the affine Artin groups as fundamental groups of such spaces. Below, we will briefly review his result. 
With the notation from Section 1.1 let

$$
V=\mathfrak{h}^{*} \text {. }
$$

The finite Weyl group acts properly discontinuously on $V$ and in consequence the diagonal action of $\stackrel{\circ}{W}$ on the domain $V+i V$ has the same property (here $i=\sqrt{-1}$ ).

For $\beta \in R$ any root and any integer $k$ denote by

$$
\bar{H}_{A_{\beta}, k}=\left\{v \in V \mid\left(v, A_{\beta}\right)=k\left(A_{\beta}, A_{\beta}\right)\right\} .
$$

Consider the domain

$$
\bar{\Omega}=V+i V
$$

and the following action of $W$ on it:

- $s_{j}\left(v_{1}+i v_{2}\right)=s_{j}\left(v_{1}\right)+i s_{j}\left(v_{2}\right)$ for $j \neq 0$;

- $\lambda_{\mu}\left(v_{1}+i v_{2}\right)=v_{1}+\mu+i v_{2}$ for $\mu \in M$.

Consider the space

$$
\overline{\mathcal{Y}}:=\left((V+i V)-\bigcup_{\substack{\beta \in R \\ k \in \mathbb{Z}}}\left(\bar{H}_{A_{\beta}, k}+i \bar{H}_{A_{\beta}, 0}\right)\right)
$$

and the orbits space associated to the above action

$$
\overline{\mathcal{X}}=\overline{\mathcal{Y}} / W .
$$

Let $\bar{p}: \overline{\mathcal{Y}} \rightarrow \overline{\mathcal{X}}$ denote the canonical projection. Fix $\bar{c} \in V$ such that $\bar{c}$ is in the fundamental chamber for the action of $W$. We can choose

$$
\bar{\star}=\bar{p}(i \bar{c})
$$

as a base point for $\overline{\mathcal{X}}$. Such orbits spaces occur as the complement of the discriminant of the semi-universal deformation of simply elliptic and cusp singularities [Lo, Chapter III] and their fundamental group becomes the monodromy group of this type of singularities. The computation of the fundamental group was done by van der Lek [L2, Chapter 3, Theorem 2.5]. Before stating his result we need the following notation

- $\bar{\Upsilon}_{j}:[0,1] \rightarrow \overline{\mathcal{Y}} ; \bar{\Upsilon}_{j}(t)=\bar{c}+\frac{\mathrm{e}^{-\pi i t}-1}{2}\left(\bar{c}, \alpha_{j}^{\vee}\right) \alpha_{j}, 1 \leqslant j \leqslant n$;

- $\bar{y}_{A_{j}}:[0,1] \rightarrow \overline{\mathcal{Y}} ; \bar{y}_{A_{j}}(t)=\bar{c}+t A_{j}, 1 \leqslant j \leqslant n$.

Note that, $\bar{p} \circ \bar{\Upsilon}_{j}$ and $\bar{p} \circ \bar{y}_{A_{j}}$ are closed paths in $\overline{\mathcal{X}}$. Now, Theorem 2.5 in [L2, Chapter 3] states as follows.

THEOREM 1.11. With the notation above, the fundamental group $\pi_{1}(\overline{\mathcal{X}}, \bar{\star})$ and the affine Artin group $\mathcal{A}_{W}$ are isomorphic. Under the isomorphism the homotopy classes of the paths $\bar{p} \circ \bar{\Upsilon}_{j}$, and $\bar{p} \circ \bar{y}_{A_{j}}$ correspond to $T_{j}$ and $Y_{A_{j}}$, respectively. 
In fact, van der Lek's result is more general. It can be stated for an arbitrary finite Coxeter group. Although the statements don't include the eventual existence of imaginary roots in the root system associated with this Coxeter group, once one appropriately modifies the regular orbits space the proofs work exactly the same way to produce a corresponding result. In Section 3 we will define the space which has the fundamental group isomorphic with the double affine Artin group.

\section{The Involution}

In this section we will define an involution $\imath$ on the set with elements of the form $\left(A,\left\{\alpha_{j}\right\}\right)$ where $A$ is a irreducible affine Cartan matrix satisfying our convention in Section 1.2 and $\left\{\alpha_{j}\right\}$ is a basis of the corresponding root system.

Because the irreducible root systems are classified by their Dynkin diagrams it is enough to make precise a basis of $R^{l}$, which we denote by $\left\{\alpha_{j}^{l}\right\}_{0 \leqslant j \leqslant n}$ and the scalar products between the simple roots. The fact that we want our involution to preserve the affine type allows us to specify only $\left\{\alpha_{j}^{l}\right\}_{1 \leqslant j \leqslant n}$. For $1 \leqslant j \leqslant n$, define

$$
\alpha_{j}^{l}=\frac{A_{j}}{\sqrt{p}}
$$

the scalar product being the canonical one in $\mathfrak{h}^{*}$. It will cause no confusion we will realize the root system $R^{l}$ on $\mathfrak{h}^{*}$. Also,

$$
\delta^{l}=\delta \quad \text { and } \quad \Lambda_{0}^{l}=\Lambda_{0} .
$$

The finite Weyl groups associated to $\stackrel{\circ}{R}$ and $\stackrel{\circ}{R}^{l}$ coincide and we will not distinguish between them. The affine and double affine Weyl groups associated with $R^{l}$ we denote by $W^{\imath}$, respectively $\widetilde{W}^{\imath}$.

At the level of affine root systems the involution fixes all irreducible affine Cartan matrices, except $B_{n}^{(1)}$ and $C_{n}^{(1)}$ which are interchanged. At the level of roots the involution acts as identity for the all root systems except $B_{n}^{(1)}, C_{n}^{(1)}, F_{4}^{(1)}$ and $G_{2}^{(1)}$. For root systems of these types these the involution interchanges short and long roots. At the level of double affine Weyl groups the following result holds.

PROPOSITION 2.1. With the notation above $M^{l}=\sqrt{p} \stackrel{\circ}{Q}$ and $\stackrel{\circ}{Q}^{l}=\frac{1}{\sqrt{p}}$ M. Furthermore, the map

$$
\phi_{\widetilde{W}}: \widetilde{W} \rightarrow \widetilde{W}^{l}
$$

defined as follows

$$
\begin{array}{ll}
\phi \widetilde{\sim}(\stackrel{\circ}{w})=\stackrel{\circ}{w} & \text { for } \quad \stackrel{\circ}{w} \in \stackrel{\circ}{W}, \\
\phi_{\widetilde{W}}^{\sim}\left(\lambda_{\mu}\right)=\tau_{\frac{1}{\sqrt{p}}}^{l} \mu & \text { for } \quad \mu \in M, \\
\phi_{\widetilde{W}}\left(\tau_{\beta}\right)=\lambda_{\sqrt{p} \beta}^{l} & \text { for } \quad \beta \in \stackrel{\circ}{Q}, \\
\phi_{\widetilde{W}}^{\sim}\left(\tau_{\delta}\right)=\tau_{-\delta}^{l} . &
\end{array}
$$


is an isomorphism of groups. The inverse of $\phi_{\widetilde{W}}$ is $\phi_{\widetilde{W}^{1}}$.

Proof. All computations are straightforward.

The main results of the paper state that the isomorphism between the double affine Weyl groups from the previous proposition induces isomorphisms at the level of double affine Artin groups and double affine Hecke algebras. In order to keep our notation as simple as possible we denoted all these isomorphisms by the same symbol.

THEOREM 2.2. The map

$$
\begin{aligned}
& \phi_{\widetilde{W}}\left(T_{j}\right)=T_{j}^{l-1} \quad \text { for } \quad 1 \leqslant j \leqslant n, \\
& \phi_{\widetilde{W}}\left(Y_{\mu}\right)=X_{\frac{1}{\sqrt{p}} \mu}^{l} \text { for } \quad \mu \in M, \\
& \phi_{\widetilde{W}}\left(X_{\beta}\right)=Y_{\sqrt{p} \beta}^{l} \quad \text { for } \quad \beta \in \stackrel{\circ}{Q}, \\
& \phi_{\widetilde{W}}\left(X_{\delta}\right)=X_{-\delta}^{l},
\end{aligned}
$$

can be uniquely extended to an isomorphism

$$
\phi_{\widetilde{W}}: \mathcal{A}_{\widetilde{W}} \rightarrow \mathcal{A}_{\widetilde{W}^{\prime}},
$$

between the double affine Artin groups. The inverse of $\phi_{\widetilde{W}}$ is $\phi_{\widetilde{W}^{i}}$.

THEOREM 2.3. The group isomorphism from Theorem 2.2 extended C-linearly and by

$$
\begin{aligned}
& \phi \widetilde{W}\left(t_{j}\right)=t_{j}^{-1} \quad \text { for } j \neq 0, \\
& \phi_{\widetilde{W}}(q)=q^{-1} .
\end{aligned}
$$

induces an isomorphism between the corresponding Hecke algebras. The inverse of $\phi_{\widetilde{W}}$ is $\phi_{\widetilde{W}^{\prime}}$.

The proofs of Theorem 2.2 and Theorem 2.3 will be concluded in Section 3 and Section 4 , respectively.

\section{The Topological Interpretation}

\subsection{THE ORBITS SPACE}

The proof of Theorem 2.2 relies on the interpretation of the double affine Artin group $\mathcal{A}_{\widetilde{W}}$ as a fundamental group of a certain topological space. In what follows we will present the construction of this space.

With the notation from Section 1.1 let

$$
V=\mathfrak{h}^{*} \quad \text { and } \quad \tilde{V}=\mathfrak{h}^{*}+\mathbb{R} \Lambda_{0} .
$$

The Tits cone is defined to be

$$
I:=\bigcup_{w \in W} w(\bar{C})
$$


where

$$
C:=\left\{\tilde{v} \in \tilde{V} \mid\left(\tilde{v}, \alpha_{j}\right)>0,0 \leqslant j \leqslant n\right\}
$$

is the fundamental chamber for the action of the affine Weyl group $W$. The interior of the Tits cone is

$$
\stackrel{\circ}{I}=\left\{v+r \Lambda_{0} \mid v \in V, r>0\right\} .
$$

Recall that $x \in I$ is an interior point if and only if $\operatorname{stab}_{W}(x)$ is a finite group. The affine Weyl group acts properly discontinuously on $I$ and in consequence the diagonal action of $W$ on the domain $\tilde{V}+i \stackrel{\circ}{I}$ has the same property.

For $\beta \in R^{r e}$ any real root (that is, nonproportional with the imaginary root $\delta$ ) and any integer $k$ denote by

$$
\tilde{H}_{\beta, k}=\left\{\tilde{v} \in \tilde{V} \mid\left(\tilde{v}, \beta^{\vee}\right)=k\right\} .
$$

Consider the domain

$$
\tilde{\Omega}=(\tilde{V}+i \stackrel{\circ}{I}) \times \mathbb{C}
$$

and the following action of $\widetilde{W}$ on it:

- $s_{j}\left(\tilde{v}_{1}+i \tilde{v}_{2} ; z\right)=\left(s_{j}\left(\tilde{v}_{1}\right)+i s_{j}\left(\tilde{v}_{2}\right) ; z\right)$ for $j \neq 0$;

- $s_{0}\left(\tilde{v}_{1}+i \tilde{v}_{2} ; z\right)=\left(\tilde{v}_{1}+i \tilde{v}_{2}+\left(\tilde{v}_{1}+i \tilde{v}_{2}, \alpha_{0}\right) \theta ; z \mathrm{e}^{\frac{\pi i}{2}\left\{\left(\tilde{v}_{1}, \theta\right)-\frac{\left(\tilde{v}_{1}, \delta\right)}{\left(\hat{v}_{2}, \delta\right)}\left(\tilde{v}_{2}, \theta\right)\right\}}\right)$;

- $\tau_{\beta}\left(\tilde{v}_{1}+i \tilde{v}_{2} ; z\right)=\left(\tilde{v}_{1}+\beta+i \tilde{v}_{2} ; z \mathrm{e}^{\frac{\pi i}{2\left(\tilde{v}_{2}, \beta\right)}}\right)$ for $\beta \in \stackrel{\circ}{Q}$;

- $\tau_{\delta}\left(\tilde{v}_{1}+i \tilde{v}_{2} ; z\right)=\left(\tilde{v}_{1}+i \tilde{v}_{2},-z\right)$.

Consider the following space

$$
\tilde{\mathcal{Y}}:=\left((\tilde{V}+i \stackrel{\circ}{I})-\bigcup_{\substack{\beta \in R^{r e} \\ k \in Z}}\left(\tilde{H}_{\beta, k}+i \tilde{H}_{\beta, 0}\right)\right) \times \mathbb{C}^{*}
$$

and the orbits space associated to the above action $\tilde{\mathcal{X}}=\tilde{\mathcal{Y}} / \tilde{W}$. Let $\tilde{p}: \tilde{\mathcal{Y}} \rightarrow \tilde{\mathcal{X}}$ denote the canonical projection. Fix $c \in V$ such that $c+\Lambda_{0}$ is in the fundamental chamber and the numbers $\left\{\left(c, \alpha_{j}\right)\right\}_{1 \leqslant j \leqslant n}$ are positive and sufficiently small. Fix also $z_{0}$ a positive real number and let $u=c+i c$. Then the point $\left(u+i \Lambda_{0} ; z_{0}\right)$ is in $\tilde{\mathcal{Y}}$ and we can choose $\tilde{\star}=\tilde{p}\left(u+i \Lambda_{0} ; z_{0}\right)$ as a base point for $\tilde{\mathcal{X}}$. To state Theorem 2.5 in [L2, Section 3] adapted to our present situation we need the following notation

- $\tilde{\Upsilon}_{j}:[0,1] \rightarrow \tilde{\mathcal{Y}} ; \tilde{\Upsilon}_{j}(t)=\left(u+i \Lambda_{0}+\frac{\mathrm{e}^{\pi i t}-1}{2}\left(u, \alpha_{j}^{\vee}\right) \alpha_{j} ; z_{0}\right), 1 \leqslant j \leqslant n$;

- $\tilde{\Upsilon}_{0}:[0,1] \rightarrow \tilde{\mathcal{Y}} ; \tilde{\Upsilon}_{0}(t)=\left(u+i \Lambda_{0}-\frac{\mathrm{e}^{\frac{\pi}{\pi t}}-1}{2}\left(u+i \Lambda_{0}, \alpha_{0}\right) \theta ; z_{0} \mathrm{e}^{\frac{\pi i t}{2}(c, \theta)}\right)$;

- $\tilde{x}_{\alpha_{j}}:[0,1] \rightarrow \tilde{\mathcal{Y}} ; \tilde{x}_{\alpha_{j}}(t)=\left(u+i \Lambda_{0}+t \alpha_{j} ; z_{0} \mathrm{e}^{\frac{\pi i t}{2}(c, \beta)}\right), 1 \leqslant j \leqslant n$;

- $\tilde{x}_{\delta}:[0,1] \rightarrow \tilde{\mathcal{Y}} ; \tilde{x}_{\delta}(t)=\left(u+i \Lambda_{0} ; z_{0} \mathrm{e}^{\pi i t}\right)$.

Note that $\tilde{p} \circ \tilde{\Upsilon}_{j}, \tilde{p} \circ \tilde{x}_{\alpha_{j}}$ and $\tilde{p} \circ \tilde{x}_{\delta}$ are closed paths in $\tilde{\mathcal{X}}$. Now, van der Lek's result for the affine Weyl group reads as follows. 
THEOREM 3.1. With the notation above, we have an isomorphism between the fundamental group $\pi_{1}(\tilde{\mathcal{X}}, \tilde{\star})$ and the double affine Artin group $\mathcal{A}_{\tilde{W}}$. Under the isomorphism the homotopy classes of the paths $\tilde{p} \circ \tilde{\Upsilon}_{j}, \tilde{p} \circ \tilde{x}_{\alpha_{j}}$ and $\tilde{p} \circ \tilde{x}_{\delta}$ correspond to $T_{j}, X_{\alpha_{j}}$ and $X_{\delta}$, respectively.

What will be crucial in the proof of Theorem 2.2 is another presentation of the double affine Artin group as a fundamental group of a slightly different topological space. Consider the subspace

$$
\Omega=(V+i V) \times \mathrm{C} \hookrightarrow \tilde{\Omega} ; \quad\left(v_{1}+i v_{2} ; z\right) \mapsto\left(v_{1}+i v_{2}+i \Lambda_{0} ; z\right) .
$$

It is a simple fact that $\Omega$ is invariant under the action of $\widetilde{W}$. Let us make this action explicit:

- $s_{j}\left(v_{1}+i v_{2} ; z\right)=\left(s_{j}\left(v_{1}\right)+i s_{j}\left(v_{2}\right) ; z\right)$ for $j \neq 0$;

- $s_{0}\left(v_{1}+i v_{2} ; z\right)=\left(s_{\theta}\left(v_{1}\right)+i s_{\theta}\left(v_{2}\right)+i \theta ; z \mathrm{e}^{\frac{\pi i}{2}\left(v_{1}, \theta\right)}\right)$;

- $\lambda_{\mu}\left(v_{1}+i v_{2} ; z\right)=\left(v_{1}+i v_{2}+i \mu ; z \mathrm{e}^{-\frac{\pi}{2}\left(v_{1}, \mu\right)}\right)$ for $\mu \in M$;

- $\tau_{\beta}\left(v_{1}+i v_{2} ; z\right)=\left(v_{1}+\beta+i v_{2} ; z \mathrm{e}^{\frac{\pi i}{2}\left(v_{2}, \beta\right)}\right)$ for $\beta \in \mathrm{Q}$;

- $\tau_{\delta}\left(v_{1}+i v_{2} ; z\right)=\left(v_{1}+i v_{2},-z\right)$.

For any nonzero $\beta \in V$ and $k \in Z$ define the hyperplanes

$$
H_{\beta, k}:=\left\{v \in V \mid \frac{2(v, \beta)}{(\beta, \beta)}=k\right\} .
$$

As before, we can consider the space

$$
\mathcal{Y}:=\left((V+i V)-\bigcup_{\substack{\beta \in R^{\circ} \\ h, k \in \bar{Z}}}\left(H_{\beta, h}+i H_{A_{\beta}, k}\right)\right) \times \mathbb{C}^{*},
$$

the orbits space $\mathcal{X}=\mathcal{Y} / \widetilde{W}$ and the canonical projection $p: \mathcal{Y} \rightarrow \mathcal{X}$. The point $\left(u ; z_{0}\right)$ is in $\mathcal{Y}$ and we can choose $\star=p\left(u ; z_{0}\right)$ as a base point for $\mathcal{X}$.

We can define a mapret: $\tilde{\mathcal{Y}} \rightarrow \mathcal{Y}$, as follows

$$
\operatorname{ret}\left(\tilde{v}_{1}+i \tilde{v}_{2} ; z\right)=\left(\tilde{v}_{1}-\frac{\left(\tilde{v}_{1}, \delta\right)}{\left(\tilde{v}_{2}, \delta\right)} \tilde{v}_{2}+i \frac{1}{\left(\tilde{v}_{2}, \delta\right)} \tilde{v}_{2}-i \Lambda_{0} ; z\right)
$$

Simple computations show that this map is well defined.

PROPOSITION 3.2. The map ret: $\tilde{\mathcal{Y}} \rightarrow \mathcal{Y}$ defined above is a deformation retract. Moreover, ret is $\widetilde{W}$ equivariant and consequently $\tilde{\mathcal{X}}$ and $\mathcal{X}$ have the same homotopy type.

Proof. All computations are straightforward. 
Now Theorem 3.1 takes a more symmetric form. Let us define first the ingredients:

- $\Upsilon_{j}:[0,1] \rightarrow \mathcal{Y} ; \Upsilon_{j}(t)=\left(u+\frac{\mathrm{e}^{\pi i t}-1}{2}\left(u, \alpha_{j}^{\vee}\right) \alpha_{j} ; z_{0}\right), 1 \leqslant j \leqslant n$;

- $\Upsilon_{0}:[0,1] \rightarrow \mathcal{Y} ; \Upsilon_{0}(t)=\left(u-\frac{\mathrm{e}^{\frac{2 \pi t}{2}}-1}{2}\left(u+i \Lambda_{0}, \alpha_{0}\right) \theta ; z_{0} \mathrm{e}^{\frac{\pi i t}{2}(c, \theta)}\right)$;

- $y_{A_{j}}:[0,1] \rightarrow \mathcal{Y} ; y_{A_{j}}(t)=\left(u+i t A_{j} ; z_{0} \mathrm{e}^{-\frac{\pi i t}{2}\left(c, A_{j}\right)}\right), 1 \leqslant j \leqslant n$;

- $x_{\alpha_{j}}:[0,1] \rightarrow \mathcal{Y} ; x_{\alpha_{j}}(t)=\left(u+t \alpha_{j} ; z_{0} \mathrm{e}^{\frac{\pi i t}{2}\left(c, \alpha_{j}\right)}\right), 1 \leqslant j \leqslant n$;

- $x_{\delta}:[0,1] \rightarrow \mathcal{Y} ; x_{\delta}(t)=\left(u ; z_{0} \mathrm{e}^{\pi i t}\right)$.

Note that $p \circ \Upsilon_{j}, p \circ y_{A_{j}}, p \circ x_{\alpha_{j}}$ and $p \circ x_{\delta}$ are closed paths in $\mathcal{X}$.

THEOREM 3.3. With the notation above, we have an isomorphism between the fundamental group $\pi_{1}(\mathcal{X}, \star)$ and the double affine Artin group $\mathcal{A}_{\widetilde{W}}$. Under the isomorphism the homotopy classes of the paths $p \circ \Upsilon_{j}, p \circ y_{A_{j}}, p \circ x_{\alpha_{j}}$ and $p \circ x_{\delta}$ correspond to $T_{j}, Y_{A_{j}}, X_{\alpha_{j}}$ and $X_{\delta}$, respectively.

Proof. It follows from Theorem 3.1 and Proposition 3.2 that the fundamental

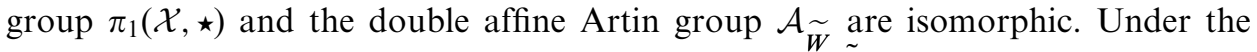
isomorphism the homotopy classes of the paths $p \circ \operatorname{ret}\left(\tilde{\Upsilon}_{j}\right)=p \circ \Upsilon_{j}($ for $0 \leqslant j \leqslant n$ ), $p \circ \operatorname{ret}\left(\tilde{x}_{\alpha_{j}}\right)=p \circ x_{\alpha_{j}}$ (for $1 \leqslant j \leqslant n$ ) and $p \circ \operatorname{ret}\left(\tilde{x}_{\delta}\right)=p \circ x_{\delta} \operatorname{correspond}$ to $T_{j}, X_{\alpha_{j}}$ and $X_{\delta}$, respectively. Moreover, by a computation very similar to the one done in the proof of Theorem 5.5 in [L2, Chapter 3] one can show that this is also an isomorphism between the subgroup of $\pi_{1}(\mathcal{X}, \star)$ generated by $p \circ y_{A_{j}}$ (for $1 \leqslant j \leqslant n$ ) and $p \circ \Upsilon_{j}$ (for $1 \leqslant j \leqslant n$ ) and the affine Artin group $\mathcal{A}_{W}$ and that $p \circ y_{A_{j}}$ correspond to $Y_{A_{j}}$ under this isomorphism.

\subsection{PROOF OF THEOREM 2.2}

Let us start by an analysis of the constructions in Section 3.1 for the group $\widetilde{W}^{l}$. Because

$$
M^{l}=\sqrt{p} \stackrel{\circ}{Q} \quad \text { and } \quad \stackrel{\circ}{Q}=\frac{1}{\sqrt{p}} M
$$

we obtain that there exists a one-to-one correspondence between the finite root systems $R$ and $\stackrel{\circ}{R}^{l}$ which assigns to any $\beta \in \stackrel{\circ}{R}$ a root $\beta^{l} \in \stackrel{\circ}{R}^{l}$ such that

$$
\beta^{l}=\frac{1}{\sqrt{p}} A_{\beta} \quad \text { and } \quad A_{\beta^{l}}=\sqrt{p} \beta .
$$

Therefore, we have that

$$
H_{\beta^{l}, k}=\frac{1}{\sqrt{p}} H_{A_{\beta}, k} \quad \text { and } \quad H_{A^{1}, h}=\sqrt{p} H_{\beta, h},
$$

and in consequence the topological space

$$
\mathcal{Y}^{l}=\left((V+i V)-\bigcup_{\substack{\beta \in R^{\circ} \\ h, k \in \mathcal{Z}}}\left(\frac{1}{\sqrt{p}} H_{A_{\beta}, k}+i \sqrt{p} H_{\beta, h}\right)\right) \times \mathbb{C}^{*} .
$$


If we denote by

$$
\mathcal{Z}=\left((V+i V)-\bigcup_{\substack{\beta \in \mathcal{R}^{\circ} \\ h, k \in \mathcal{Z}}}\left(H_{A_{\beta}, k}+i H_{\beta, h}\right)\right) \times \mathbb{C}^{*},
$$

we see that the map

$$
\mathcal{Y}^{l} \rightarrow \mathcal{Z}, \quad\left(v_{1}+i v_{2} ; z\right) \mapsto\left(\sqrt{p} v_{1}+i \frac{1}{\sqrt{p}} v_{2} ; z\right)
$$

is a homeomorphism. We can push forward the action of $\widetilde{W}^{l}$ obtaining the following formulas for the action on $\mathcal{Z}$ :

- $s_{j}^{l}\left(v_{1}+i v_{2} ; z\right)=\left(s_{j}\left(v_{1}\right)+i s_{j}\left(v_{2}\right) ; z\right)$ for $j \neq 0$;

- $\lambda_{\sqrt{p} \beta}^{l}\left(v_{1}+i v_{2} ; z\right)=\left(v_{1}+i v_{2}+i \beta ; z e^{-\frac{\pi i}{2}\left(v_{1}, \beta\right)}\right)$ for $\beta \in \stackrel{\circ}{Q}$;

- $\tau_{\frac{1}{\sqrt{1}} \mu}^{\nu}\left(v_{1}+i v_{2} ; z\right)=\left(v_{1}+\mu+i v_{2} ; z e^{\frac{\pi}{2}\left(v_{2}, \mu\right)}\right)$ for $\mu \in M$;

- $\tau_{\delta}^{\sqrt{p}}\left(v_{1}+i v_{2} ; z\right)=\left(v_{1}+i v_{2},-z\right)$.

By Theorem 3.3 and the above considerations the double affine Artin group $\mathcal{A}_{\widetilde{W}}$ is isomorphic to the fundamental group of $\mathcal{X}^{\prime}$, the orbits space associated with $\mathcal{Z}$ and the above action of $\widetilde{W}^{l}$. To make this precise let us define the following paths on $\mathcal{Z}$ :

- $\Upsilon_{j}^{l}:[0,1] \rightarrow \mathcal{Z} ; \Upsilon_{j}^{l}(t)=\left(u+\frac{e^{\pi i t}-1}{2}\left(u, \alpha_{j}^{\vee}\right) \alpha_{j} ; z_{0}\right), 1 \leqslant j \leqslant n$;

- $y_{\sqrt{p} \alpha_{j}}^{l}:[0,1] \rightarrow \mathcal{Z} ; y_{\sqrt{p} \alpha_{j}}^{l}(t)=\left(u+i t \alpha_{j} ; z_{0} e^{-\frac{\pi i t}{2}\left(c, \alpha_{j}\right)}\right), 1 \leqslant j \leqslant n$;

- $x_{\frac{1}{\sqrt{p}} A_{j}}^{l}:[0,1] \rightarrow \mathcal{Z} ; x_{\frac{1}{\sqrt{p}} A_{j}}^{l}(t)=\left(u+t A_{j} ; z_{0} e^{\frac{\pi i t}{2}\left(c, A_{j}\right)}\right), 1 \leqslant j \leqslant n$

- $x_{\delta}^{l}:[0,1] \rightarrow \mathcal{Z} ; x_{\delta}^{l}(t)=\left(u ; z_{0} e^{\pi i t}\right)$.

By $p^{l}: \mathcal{Z} \rightarrow \mathcal{X}^{\prime}$ we denote the canonical projection. Theorem 3.3 reads now as follows.

THEOREM 3.4. With the notation above, we have an isomorphism between the fundamental group $\pi_{1}\left(\mathcal{X}^{\prime}, \star\right)$ and the double affine Artin group $\mathcal{A}_{\widetilde{W}^{2}}$. Under the isomorphism the homotopy classes of the paths $p^{l} \circ \Upsilon_{j}^{l}, p^{l} \circ y_{\sqrt{p} \alpha_{j}}^{l}, p^{l} \circ x_{\frac{1}{\sqrt{p}} A_{j}}^{l}$ and $p^{l} \circ x_{\delta}^{l}$ correspond to $T_{j}^{l}, Y_{\sqrt{p} \alpha_{j}}^{l}, X_{\frac{1}{\sqrt{p}} A_{j}}^{l}$ and $X_{\delta}^{l}$, respectively.

Looking back at the way $\mathcal{X}$ was defined we see that the map

$$
\mathcal{Y} \rightarrow \mathcal{Z}, \quad\left(v_{1}+i v_{2} ; z\right) \mapsto\left(v_{2}+i v_{1} ; \bar{z}\right),
$$

induces an isomorphism of fundamental groups of the associated orbits spaces (by $\bar{z}$ we denoted the complex conjugate of $z$ ). A straightforward analysis shows that the homotopy classes of the paths $p \circ \Upsilon_{j}, p \circ y_{A_{j}}, p \circ x_{\alpha_{j}}, p \circ x_{\delta}$ and $\left(p^{l} \circ \Upsilon_{j}^{l}\right)^{-1}, p^{l} \circ x_{\frac{1}{\sqrt{p}} A_{j}}^{l}$, $p^{l} \circ y_{\sqrt{p} \alpha_{j}}^{l},\left(p^{l} \circ x_{\delta}^{l}\right)^{-1}$ correspond respectively. Combining this with the Theorem 3.3 and Theorem 3.4 we finish our proof of Theorem 2.2. 


\section{Descent to Hecke Algebras}

\subsection{SOME COMBINATORIAL RESULTS}

Let us first establish some notation. For each $w$ in $W$ let $l(w)$ be the length of a reduced (i.e. shortest) decomposition of $w$ in terms of the $s_{j}$. We have

$$
l(w)=|\Pi(w)|,
$$

where

$$
\Pi(w)=\left\{\alpha \in R_{+} \mid w(\alpha) \in R_{-}\right\} .
$$

If $w=s_{j_{p}} \cdots s_{j_{1}}$ is a reduced decomposition, then

$$
\Pi(w)=\left\{\alpha^{(k)} \mid 1 \leqslant k \leqslant p\right\},
$$

with $\alpha^{(k)}=s_{j_{1}} \ldots s_{j_{k-1}}\left(\alpha_{j_{k}}\right)$.

For the basic properties of the length function on Coxeter groups see [H]. Let us list the most important ones:

(1) For each $0 \leqslant j \leqslant n$ we have $l\left(s_{j} w\right)=l(w) \pm 1$;

(2) If $l\left(s_{j} w\right)=l(w)-1$ then $s_{j} w$ can be obtained from a certain reduced decomposition of $w$ by omitting a $s_{j}$ factor.

When $w \in W$ can be written as $\stackrel{\circ}{w} \lambda_{\mu}$, with $\stackrel{\circ}{w} \in \stackrel{\circ}{W}$ and $\mu \in M$ the formula (10) takes the following form (see $[\mathrm{Lu}]$ )

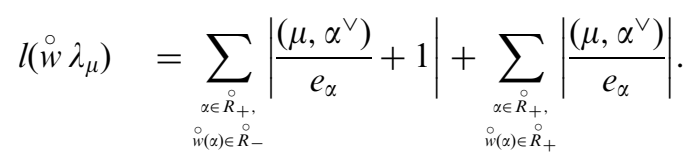

Next, we will consider an application of the above formula. In this subsection we will suppose that our irreducible affine Cartan matrix satisfies the restriction imposed in Section 1.2 and moreover it is such that $p \neq 1$. Precisely in this case $\theta$ is the highest root of the associated finite root system and it is not equal to $\theta_{s}$ the highest short root of the associated finite root system.

LEMMA 4.1. Let $\mathcal{A}_{W}$ be the Artin group associated to an irreducible affine Cartan matrix as above. Then, in $\mathcal{A}_{W}$ we have $Y_{-\theta_{s}^{\vee}}=T_{s_{\theta_{s}}} T_{0} T_{s_{\theta-\theta_{s}}} T_{0}$.

Proof. First, let us see that the formula in the statement makes sense. When $p \neq 1$ each $e_{\alpha}$ is equal to $d_{\alpha}$, therefore $e_{\alpha}^{-1} \alpha^{\vee}=\alpha$ and $M=\stackrel{\circ}{Q}^{\vee}$. In consequence $-\theta_{s}^{\vee}$ is an anti-dominant element of $M$. Moreover, using basic facts about root systems, which can be found for example in [B, VI, Sections 1,3$]$, we obtain that $\left(\theta, \theta_{s}\right)=1$, which implies that $\theta-\theta_{s}=s_{\theta}\left(-\theta_{s}\right) \in R$. Denote by $w=s_{0} s_{\theta-\theta_{s}} s_{0}$. A simple computation shows that $s_{\theta_{s}} w=\lambda_{-\theta_{s}^{v}}$. In the view of Remark 1.4 our statement follows from the following formulas

$$
l(w)=l\left(s_{\theta-\theta_{s}}\right)+2
$$


and

$$
l(w)+l\left(s_{\theta_{s}}\right)=l\left(\lambda_{-\theta_{s}}\right) .
$$

The Equation (13) immediately follows from the second property of the length function mentioned above keeping in mind that $w \notin W$, fact which can be easily checked. Writing down formula (12) for $\lambda_{-\theta_{s}^{\vee}}, s_{\theta_{s}}$ and for $w=s_{\theta_{s}} \lambda_{-\theta_{s}^{\vee}}$ we obtain

$$
\begin{aligned}
& l\left(\lambda_{-\theta_{s}^{\vee}}\right)=\sum_{\substack{\in \in R_{+},\left(\alpha, \theta_{s}\right) \neq 0}}\left(\theta_{s}^{\vee}, \alpha\right), \\
& l\left(s_{\theta_{s}}\right)=\sum_{\substack{\alpha \in R_{+},\left(\alpha, \theta_{s}^{\vee}\right) \neq 0}} 1, \\
& l\left(s_{\theta_{s}} \lambda_{-\theta_{s}^{\vee}}\right)=\sum_{\substack{\alpha \in R_{+}^{\circ},\left(\alpha, \theta_{s}^{\vee} \neq 0\right.}}\left\{\left(\theta_{s}^{\vee}, \alpha\right)-1\right\} .
\end{aligned}
$$

These formulas show that Equation (14) holds. The proof statement is completed.

The following fact will be crucial.

LEMMA 4.2. With the notation above we have

$$
\Pi\left(s_{\theta-\theta_{s}}\right)-\left\{\theta-\theta_{s}\right\} \subseteq\left\{\alpha \in \stackrel{\circ}{R_{+}} \mid\left(\alpha^{\vee}, \theta-\theta_{s}\right)=1\right\} .
$$

Proof. In the case when $p=3$, the matrix $A$ equals $G_{2}$, and our statement can be checked. Indeed, if $\alpha_{1}, \alpha_{2}$ is the standard basis, with $\alpha_{1}$ the short root and $\alpha_{2}$ the long root, we have that $\theta=3 \alpha_{1}+2 \alpha_{2}, \theta_{s}=2 \alpha_{1}+\alpha_{2}$ and $\theta-\theta_{s}=\alpha_{1}+\alpha_{2}$. Furthermore, $s_{\theta-\theta_{s}}=s_{2} s_{1} s_{2}$ is a reduced decomposition and

$$
\Pi\left(s_{\theta-\theta_{s}}\right)=\left\{\alpha_{1}+\alpha_{2}, \alpha_{2}, 3 \alpha_{1}+2 \alpha_{2}\right\} .
$$

Keeping in mind that

$$
\left(\alpha_{1}, \alpha_{1}\right)=2 / 3, \quad\left(\alpha_{1}, \alpha_{2}\right)=-1 \quad \text { and } \quad\left(\alpha_{2}, \alpha_{2}\right)=2,
$$

the conclusion follows.

When $p=2$, it is well known (see [B, VI, Sections 1, 3]) that we have the following possible values for the scalar products $\left(\alpha, \beta^{\vee}\right)$ for any roots $\alpha, \beta$ such that $\alpha \neq \pm \beta$ :

1. if $\beta$ is long, $\left(\alpha, \beta^{\vee}\right) \in\{0, \pm 1\}$;

2. if $\beta$ and $\alpha$ are short, $\left(\alpha, \beta^{\vee}\right) \in\{0, \pm 1\}$;

3. if $\beta$ is short and $\alpha$ is long, $\left(\alpha, \beta^{\vee}\right) \in\{0, \pm 2\}$.

Moreover, $(\alpha, \alpha) \in\{1,2\}$.

Obviously, $\theta-\theta_{s} \in \Pi\left(s_{\theta-\theta_{s}}\right)$. Let $\alpha \in \Pi\left(s_{\theta-\theta_{s}}\right), \alpha \neq \theta-\theta_{s}$. Then,

$$
\left(s_{\theta-\theta_{s}}(\alpha), \theta_{s}\right)=\left(\alpha, \theta_{s}\right) \quad \text { and } \quad\left(s_{\theta-\theta_{s}}(\alpha), \theta\right)=\left(\alpha, 2 \theta_{s}-\theta\right) .
$$


Because $\alpha$ is a positive root, and $s_{\theta-\theta_{s}}(\alpha)$ is a negative root the first scalar product must be zero. This implies that the second scalar product equals $-(\alpha, \theta)$. This one cannot be zero because it would follow that $\alpha$ is fixed by $s_{\theta-\theta_{s}}$. Furthermore, $\alpha \neq \theta$ because $\left(\theta, \theta_{s}\right)=1 \neq 0$. Now, the above considerations on the possible values of scalar products show that $(\alpha, \theta)=1$. Considering all these facts we obtain $\left(\alpha,\left(\theta-\theta_{s}\right)^{\vee}\right)=2$. Keeping in mind that $\theta-\theta_{s}$ is a short root different from $\alpha$, the same considerations on scalar products imply that $\alpha$ is a long root, and consequently $\left(\alpha^{\vee}, \theta-\theta_{s}\right)=1$.

We can choose a reduced decomposition for $s_{\theta-\theta_{s}}$ of the form

$$
s_{j_{p}} \ldots s_{j_{1}} s_{j_{0}} s_{j_{1}} \ldots s_{j_{p}}
$$

with $w=s_{j_{1}} \ldots s_{j_{p}}$ the minimal length element of $\stackrel{\circ}{W}$ for which $w\left(\theta-\theta_{s}\right)=\alpha_{j_{0}}$ is a simple (short) root. Then, using formula (11) we see that $\Pi(w) \subseteq \Pi\left(s_{\theta-\theta_{s}}\right)-\left\{\theta-\theta_{s}\right\}$. As before denote $\Pi(w)=\left\{\alpha^{(k)} \mid 1 \leqslant k \leqslant p\right\}$, where the positive root $\alpha^{(k)}=s_{j_{p}} \ldots s_{j_{k-1}}\left(\alpha_{j_{k}}\right)$. By Lemma 4.2 we obtain that $\left(\theta-\theta_{s},\left(\alpha^{(k)}\right)^{\vee}\right)=1$, or equivalently

$$
\left(s_{j_{k+1}} \ldots s_{j_{p}}\left(\theta-\theta_{s}\right), \alpha_{j_{k}}^{\vee}\right)=1 .
$$

LEMMA 4.3. With the notation above, the relation $T_{w^{-1}}^{-1} X_{\theta-\theta_{s}}=X_{\alpha_{j_{0}}} T_{w}$ holds in the Artin group $\mathcal{A}_{\widetilde{W}}$.

Proof. Using the formula (16) and the relations in the double affine Artin group we obtain that for all $p \geqslant k \geqslant 1$

$$
T_{\alpha_{j_{k}}}^{-1} X_{s_{j_{k+1}} \ldots s_{j p}\left(\theta-\theta_{s}\right)}=X_{s_{j_{k}} \ldots s_{j p}\left(\theta-\theta_{s}\right)} T_{\alpha_{j_{k}}} .
$$

Now, our conclusion follows by applying these formulas.

PROPOSITION 4.4. Let $\mathcal{H}_{\widetilde{W}}$ be the double affine group associated to an irreducible affine Cartan matrix as before. Then, in $\mathcal{H}_{\widetilde{W}}$ we have

$$
T_{\theta-\theta_{s}}^{-1} X_{\theta-\theta_{s}}-\left(T_{\theta-\theta_{s}}^{-1} X_{\theta-\theta_{s}}\right)^{-1}=\frac{1}{t_{s}^{2}}-t_{s}^{-\frac{1}{2}} .
$$

Proof. From (15) we get that $T_{\theta-\theta_{s}}=T_{w^{-1}} T_{j_{0}} T_{w}$. Therefore,

$$
\begin{aligned}
T_{\theta-\theta_{s}}^{-1} X_{\theta-\theta_{s}} & =T_{w}^{-1} T_{j_{0}}^{-1} T_{w^{-1}}^{-1} X_{\theta-\theta_{s}} \\
& =T_{w}^{-1} T_{j_{0}}^{-1} X_{\alpha_{j_{0}}} T_{w} \\
& =T_{w}^{-1}\left(X_{-\alpha_{j_{0}}} T_{j_{0}}+\left(t_{s}^{\frac{1}{2}}-t_{s}^{-\frac{1}{2}}\right)\right) T_{w} \\
& =X_{-\theta+\theta_{s}} T_{w^{-1}} T_{j_{0}} T_{w}+\left(t_{s}^{\frac{1}{2}}-t_{s}^{-\frac{1}{2}}\right) \\
& =\left(T_{\theta-\theta_{s}}^{-1} X_{\theta-\theta_{s}}\right)^{-1}+\left(t_{s}^{\frac{1}{2}}-t_{s}^{-\frac{1}{2}}\right) .
\end{aligned}
$$

We have used Lemma 4.3 and the fact that $\alpha_{j_{0}}$ is short. 


\subsection{PROOF OF THEOREM 2.3}

Because we already have an isomorphism at the level of Artin groups all we need to prove is that the relations (4) (5) (6) and (7) are satisfied. In order to keep the computations in $\mathcal{H}_{\widetilde{W}}$ we will check the relations for $\phi_{\widetilde{W}^{\prime}}\left(T_{j}^{l}\right), \phi_{\widetilde{W}^{\prime}}\left(T_{j}^{l} Y_{A_{j}^{l}}^{l}\right)$ and $\phi_{\widetilde{W}^{\prime}}\left(X_{-\alpha_{j}^{l}}^{l} T_{j}^{l}\right)$ (the corresponding relations for $\phi_{\widetilde{W}}\left(T_{j}\right), \phi_{\widetilde{W}}\left(T_{j} Y_{A_{j}}\right)$ and $\phi_{\widetilde{W}}\left(X_{-\alpha_{j}} T_{j}\right)$ could be checked in the same way). For $j \neq 0$ this is straightforward. For $j=0$ we have to show that

$$
\phi_{\widetilde{W}^{\prime}}\left(X_{-\alpha_{0}^{l}}^{l} T_{0}^{l}\right)-\phi_{\widetilde{W}^{\prime}}\left(X_{-\alpha_{0}^{l}}^{l} T_{0}^{l}\right)^{-1}=\phi_{\widetilde{W}^{\prime}}\left(t_{0}\right)^{-\frac{1}{2}}-\phi_{\widetilde{W}^{\prime}}\left(t_{0}\right)^{\frac{1}{2}} .
$$

There are two possible situations:

Case 1. If $p=1$, then $\alpha_{j}^{l}=\alpha_{j}$ for all $1 \leqslant j \leqslant n$. This implies that

$$
\begin{aligned}
\phi_{\widetilde{W}^{\prime}}\left(X_{-\alpha_{0}^{l}}^{l} T_{0}^{l}\right) & =\phi_{\widetilde{W}^{\prime}}\left(q X_{\theta^{\prime}}^{l}\left(T_{s_{\theta}}^{l}\right)^{-1} Y_{-\theta^{l}}^{l}\right) \\
& =q^{-1} Y_{\theta} T_{s_{\theta}} X_{-\theta} \\
& =T_{0}^{-1} X_{\alpha_{0}} .
\end{aligned}
$$

Therefore in this case the relation (18) becomes precisely the relation (7).

Case 2. If $p \neq 1$, then $\alpha_{j}^{l}=\frac{\alpha_{j}^{v}}{\sqrt{p}}$ for all $1 \leqslant j \leqslant n$. As a consequence, with the notation in Section 4.1 we have that

$$
\theta^{l}=\frac{\theta_{s}^{\vee}}{\sqrt{p}}=\sqrt{p} \theta_{s} \quad \text { and } \quad \theta_{s}^{l}=\frac{\theta}{\sqrt{p}} .
$$

Also, it is clear that $s_{\theta^{l}}=s_{\theta_{s}}$ and $\phi_{\widetilde{W}^{\prime}}\left(t_{0}\right)=t_{s}$. As before

$$
\begin{aligned}
\phi_{\widetilde{W}^{l}}\left(X_{-\alpha_{0}^{l}}^{l} T_{0}^{l}\right) & =\phi_{\widetilde{W}^{l}}\left(q X_{\theta^{\prime}}^{l}\left(T_{S_{\theta^{\prime}}}^{l}\right)^{-1} Y_{-\theta^{\prime}}^{l}\right) \\
& =q^{-1} Y_{\theta_{s}^{\vee}} T_{S_{\theta_{s}}} X_{-\theta_{s}} .
\end{aligned}
$$

Using Lemma 4.1 and the commuting relations in the double affine Artin group we obtain

$$
\phi_{\widetilde{W}^{\prime}}\left(X_{-\alpha_{0}^{l}}^{l} T_{0}^{l}\right)=T_{0}^{-1} T_{s_{\theta-\theta_{s}}}^{-1} X_{\theta-\theta_{s}} T_{0} .
$$

Conjugating (18) by $T_{0}$ we obtain the relation (17) which was proved to be true in Proposition 4.4. The proof of Theorem 2.3 is complete.

\section{Acknowledgements}

I would like to thank Professor Siddhartha Sahi for many illuminating discussions on the subject and the referee for a detailed report and for bringing to our attention the results of Koornwinder [Ko] and Macdonald [M5].

\section{References}

[B] Bourbaki, N.: Groupes et algèbres de Lie, Ch. IV, V, VI. Hermann, Paris, 1968.

[Br] Brieskorn, E.: Die Fundamentalgruppe des Raumes der regulären Orbits einer endlichen komplexen Spiegelungsgruppe, Invent. Math. 12 (1971), 57-61. 
[C1] Cherednik, I.: Double affine Hecke algebras, Knizhnik-Zamolodchikov equations, and Macdonald's operators, Internat. Math. Res. Notices (1992), No. 9, 171-180.

[C2] Cherednik, I.: Double affine Hecke algebras and Macdonald's conjectures, Ann. of Math. (2) 141(1) (1995), 191-216.

[C3] Cherednik, I.: Macdonald's evaluation conjectures and difference Fourier transform, Invent. Math. 122(1) (1995), 119-145.

[C4] Cherednik, I.: Intertwining operators of double affine Hecke algebras, Selecta Math. (N.S.) 3(4) (1997), 459-495.

[D] Deligne, P.: Les immeubles des groupes de tresses généralisés, Invent. Math. 17 (1972), 273-302.

[H] Humphreys, J. E.: Reflection Groups and Coxeter Groups, Cambridge Univ. Press, Cambridge, 1990.

[K] Kac, V. G.: Infinite Dimensional Lie Algebras, 3rd edn, Cambridge University Press, Cambridge, 1990

[Kn] Knop, F.: Integrality of two variable Kostka functions, J. Reine Angew. Math. 482 (1997), 177-189.

[KS] Knop, F. and Sahi. S.: A recursion and a combinatorial formula for Jack polynomials, Invent. Math. 128(1) (1997), 9-22.

[Ko] Koornwinder, T. H.: Self-duality for $q$-ultraspherical polynomials associated with the root system $A_{n}$. Unpublished manuscript, 1988.

[L1] van der Lek, H.: Extended Artin groups, In: Singularities, Part 2 (Arcata, Calif., 1981), Proc. Sympos. Pure Math. 40, Amer. Math. Soc., Providence, RI, 1983, pp. 117-121.

[L2] van der Lek, H.: The homotopy type of complex hyperplane complements. Ph.D. Thesis, Katholieke Universiteit Nijmegen, 1983.

[Lo] Looijenga, E.: Rational surfaces with an anti-canonical cycle, Ann. of Math. (2) 114(2) (1981), 267-322.

[Lu] Lusztig, G.: Affine Hecke algebras and their graded version, J. Amer. Math. Soc. 2(3) (1989), 599-635.

[M1] Macdonald, I. G.: Symmetric Functions and Hall Polynomials. 2nd edn, With contributions by A. Zelevinsky, Clarendon Press, Oxford, 1995.

[M2] Macdonald, I. G.: Affine Hecke algebras and orthogonal polynomials. Séminaire Bourbaki, Vol. 1994/95, Astérisque 237 (1996), Exp. No. 797, 4, 189-207.

[M3] Macdonald, I. G.: Constant term identities, orthogonal polynomials, and affine Hecke algebras, In: Proc. Internat. Congr. Math. Vol. I (Berlin, 1998). Doc. Math. 1998, Extra I, 303-317.

[M4] Macdonald, I. G.: Orthogonal polynomials associated with root systems. Sém. Lothar. Combin, 45 (2000/01), Art. B45a.

[M5] Macdonald, I. G.: Affine Hecke Algebras and Orthogomal Polynomials, Cambridge Tracts in Math 157, Cambridge Univ. Press, Cambridge, 2003.

[O] Opdam, E.: Harmonic analysis for certain representations of graded Hecke algebras, Acta Math. 175 (1995), 75-121.

[S1] Sahi, S.: Interpolation, integrality and a generalization of Macdonald's polynomials, Internat. Math. Res. Notices (1996), No. 10, 457-471.

[S2] Sahi, S.: Nonsymmetric Koornwinder polynomials and duality, Ann. of Math. (2) 150(1) (1999), 267-282.

[ST] Saito, K. and Takebayashi, T.: Extended affine root systems III. Elliptic Weyl groups, Publ. Res. Inst. Math. Sci. 33(2) (1997), 301-329.

[T] Takebayashi, T.: Double affine Hecke algebras and elliptic Hecke algebras, J. Algebra 253(2) (2002), 314-349. 